Mots. Les langages du politique

$111 \mid 2016$

Normes et usages de la langue en politique

\title{
«Le discours doit être analysé en rapport avec les dispositifs de mise en scène »
}

Entretien avec Patrick Charaudeau

"Discourse should be analysed in relation to the staging devices"

"El discurso tiene que ser traducido en relación con los dispositivos de puesta en escena»

Henri Boyer et Guy Lochard

\section{(2) OpenEdition}

\section{Journals}

Édition électronique

URL : https://journals.openedition.org/mots/22403

DOI : $10.4000 /$ mots. 22403

ISBN : 978-2-84788-836-2

ISSN : 1960-6001

Éditeur

ENS Éditions

\section{Édition imprimée}

Date de publication : 10 septembre 2016

Pagination : 131-146

ISBN : 978-2-84788-835-5

ISSN : 0243-6450

\section{Référence électronique}

Henri Boyer et Guy Lochard, « « Le discours doit être analysé en rapport avec les dispositifs de mise en scène » », Mots. Les langages du politique [En ligne], 111 | 2016, mis en ligne le 10 septembre 2018, consulté le 24 avril 2022. URL : http://journals.openedition.org/mots/22403 ; DOI : https://doi.org/ $10.4000 /$ mots. 22403 


\section{« Le discours doit être analysé en rapport avec les dispositifs de mise en scène "}

Mots. Les langages du politique : Depuis quelques années, votre production scientifique était principalement axée surle discours médiatique. Elle est maintenant centrée sur l'analyse du discours politique avec quatre ouvrages successifs. À quoi est dû ce déplacement?

Patrick Charaudeau : En effet, avec l'équipe du Centre d'analyse du discours, nous avions travaillé sur le discours publicitaire puis le discours médiatique et durant ces recherches, nous nous sommes rendu compte que tout dépendait de fait des processus de mise en scène. Or, jusqu'à présent, le discours politique était principalement analysé dans notre champ sous l'angle des mots et des idéologies. Je fais référence à l'époque où les travaux étaient avant tout centrés sur le discours de tel ou tel parti, et ceci sous la forme d'analyse de contenu ou d'analyses lexicales. Durant nos propres travaux, nous avons pris conscience que la production de sens dépend des processus de mise en scène du discours. J'ai donc eu envie d'apporter une nouvelle approche dans le champ de l'analyse du discours politique. Mon idée était de développer des études sur les processus de mise en scène et de scénographie, de contrat, de stratégie au sein de ce type de discours tout en réfléchissant aux questions de l'influence et de la propagande. C'est donc bien via les processus de mise en scène que je suis passé de la parole publique à la parole politique.

J'ai fait un constat parallèle, à savoir que le discours médiatique était, de façon dominante du moins, de l'ordre du narratif alors que le discours politique est de l'ordre de l'argumentatif (bien que combinant narratif et argumentatif). De plus, c'est l'époque, comme je le disais, où j'ai voulu réfléchir à la question de l'influence, ce qui m'a conduit à collaborer avec des chercheurs en psychologie sociale qui travaillaient sur les problèmes de persuasion. C'était donc là un espace de réflexion qui venait se superposer à celui de la rhétorique et de l'argumentation traditionnelle. Il est vrai que j'ai toujours eu des relations controversées avec les spécialistes de l'argumentation, dans la mesure où j'insistais sur la différence à opérer entre persuasion et argumentation. Comme le discours politique est bien le domaine de la persuasion, mon intérêt pour le descriptif et le narratif s'est déplacé sur le terrain de l'argumentatif, en interaction avec les autres modes discursifs. 
Mots. Les langages du politique : Mais le discours médiatique et le discours politique ne sont-ils pas de fait intriqués, le procédé de la "petite phrase » en étant le phénomène le plus significatif?

Patrick Charaudeau : En effet, et c'est la question de l'agenda médiatique qui m'a orienté dans cette direction. On se posait la fameuse question : sont-ce les médias qui dominent dans ce type de situation, ou est-ce le monde politique qui commande les médias? Et on en revenait ainsi à la question de l'influence. J'en suis venu ainsi à rencontrer les travaux de Bourdieu et sa critique, dans Ce que parler veut dire (Bourdieu, 1982), de la position linguistique à propos de l'acte de langage, déclarant que le pouvoir de celui-ci est dans le statut du sujet parlant, ce qu'il appelle le skeptron. De fait, cela l'amène à n'accorder aucun pouvoir à la parole puisque, pour lui, tout se joue dans la position sociale du sujet parlant, même si, plus tard, il est revenu sur ce point... Pour moi, il fallait donc lier la question des phrases, des «petites phrases», des formules, initialement travaillées par Jean-Pierre Faye puis par Alice Krieg-Planque, au dispositif de la parole politique pour montrer la force, l'impact de l'acte de langage lui-même. Je n'ai donc pas travaillé spécifiquement sur les «petites phrases» mais sur les effets de ce que j'ai appelé le mot-symptôme, qui pose de fait la question du quantitatif. Un mot peut avoir un effet très fort dans l'espace public sans être répété à l'envi. À l'origine, je suis linguiste et sémanticien. Mon projet était de montrer que le discours en tant que structure phrastique doit être analysé en rapport avec les dispositifs de mise en scène, et ce sont bien les médias qui m'ont fait découvrir les dispositifs de mise en scène.

Mots. Les langages du politique : Le concept de «contrat de communication» continue à occuper, dans votre théorisation du discours politique, une place centrale. Garde-t-il toute sa productivité si l'on considère la défiance croissante, la relation d'opposition de l'instance citoyenne vis-à-vis de l'autre principal protagoniste, l'instance politique?

Patrick Charaudeau : Je dirai que pour moi, ce concept a, au contraire, de plus en plus de pertinence et d'utilité. Le contrat de communication est pour moi de l'ordre du conceptuel. De fait, j'essaie de construire une phénoménologie du discours. Tout acte de langage dépend des conditions sociales de production, et ces conditions sociales de production ne renvoient pas seulement à l'idéologie. Nous n’avons pas qu'une mémoire des mots et des idées, mais aussi des situations de communication qui fabriquent des rituels socio-langagiers. J'essaie de toujours lier ces éléments. L'analyse de discours a diverses filiations : une filiation historique en rapport avec la rhétorique; une filiation dialogique qui concerne les jeux d'interdiscursivité et d'intertextualité; une filiation en relation avec la problématique de l'intersubjectivité, elle-même renforcée par les études interactionnistes. La prise en compte de toutes ces filiations m'a conduit à la conclusion que le sens ne dépend pas seulement de celui qui parle, ni de celui qui reçoit et interprète, 
mais d'un jeu de coconstruction. C'est cela qui se passe dans l'espace public. Toute déclaration politique est de fait une réaction. Mais quelle que soit l'intention de l'auteur de la déclaration, le sens qui s'imposera dans l'espace public sera le résultat d'une coconstruction avec ceux qui interprètent.

Le contrat de communication ne dit rien sur les conditions concrètes de l'échange. Il s'établit à différents niveaux et, en premier lieu, au niveau de sa finalité. Prenons l'exemple du discours publicitaire : avant même d'avoir lu le message publicitaire (son texte, son image), je reconnais qu'il relève de ce genre par l'ensemble de son dispositif. Et je peux même le reconnaître comme tel sans avoir lu son message. D'ailleurs, ce concept de contrat n'est pas sans rapport avec celui de scénographie de Dominique Maingueneau. Il est différent, toutefois. La scénographie est de l'ordre du spectacle, alors que le contrat relève de l'altérité : il n'y a pas de Je sans Tu, de production de sens sans prise en compte de l'autre. Je m'en suis expliqué dans le livre d'hommage qui lui a été fait et auquel j'ai participé.

Je me suis opposé un temps à François Jost qui défendait la notion de promesse. Le problème, avec la promesse, c'est qu'il s'agit d'un acte de parole dans lequel l'autre n'est qu'un prétexte. Alors que le contrat implique l'autre. C'est une métaphore empruntée au domaine juridique, qui souligne un rapport de reconnaissance entre les deux partenaires de l'acte de langage. C'est cela qui est déterminant pour moi.

Dans les premières études d'analyse de discours, il n'y avait guère de Je et de $T u$ : c'est la société qui parlait, un /l. Mon projet était de réintroduire l'idée que le sujet parlant est un Je-Nous, un Je-Nous qui ne peut être compris que dans l'altérité puisque c'est dans le rapport entre le Je et le Tu que se construit un Nous.

Pour en revenir au contrat du discours politique et à la question de la défiance citoyenne, j'ai précisé dans mes écrits que le rapport de force entre l'instance politique (lieu du pouvoir) et l'instance citoyenne (lieu de l'opinion) est d'influence réciproque et non symétrique, car du côté du citoyen existe ce que Derrida appelle un «droit de regard». Et la question est : ce droit de regard peut-il s'exercer effectivement? On entre dans une époque où le droit de regard est en train de changer. Le principe de la démocratie représentative est que l'on délègue le pouvoir à des représentants. Dans la démocratie athénienne, on se réunissait 48 fois par an et on pouvait exclure un délégué s’il n'était pas satisfaisant. Le droit de regard s'exerçait bien. C'était, dans une certaine mesure, un modèle de ce que maintenant on appelle la démocratie participative. Évidemment, ce mode de fonctionnement (il faut rappeler que la démocratie athénienne se déroulait sans la participation des femmes ni celle des étrangers) ne peut être reproduit dans nos sociétés contemporaines de millions d’individus. Le problème de la démocratie représentative, c'est que ce processus de délégation entraîne paradoxalement une forme de déresponsabilisation du citoyen qui peut se contenter de voter, sans exercer son droit 
de regard et de possible contestation. C'est là tout l'intérêt des comparaisons entre contextes sociohistoriques différents. De ce point de vue, l'expérience acquise lors de mes études linguistiques m'a été très précieuse. Elle conduit à distinguer deux niveaux : le niveau de la permanence des structures, qui est d'ordre conceptuel, et le niveau plus concret des configurations spécifiques.

Et donc, c'est dans ce même contrat politique de représentation démocratique (si le régime change, le contrat change) que se jouent diverses stratégies entre l'instance politique et l'instance citoyenne. Le contrat est donc une des conditions de base de l'interprétation du sens social.

Mots. Les langages du politique : Une notion tout aussi répandue dans l'analyse de discours, celle de dialogisme, est peu présente dans votre cadre conceptuel. Celle d'interdiscursivité que vous utilisez est-elle un équivalent?

Patrick Charaudeau : Je ne vois pas chez Bakhtine la notion de contrat. «Tous les mots et toutes les formes sont habités par des intentions », dit Bakhtine. J'apprécie le travail de Bakhtine, mais je ne cherche pas à me placer dans la ligne exclusive de tel ou tel théoricien. J'essaie d'y prendre ce qui me paraît le plus pertinent. Il est vrai que j'ai toujours privilégié les termes d'intertextualité et d'interdiscursivité, mais on reste bien là dans l'univers dialogique. J'ai cependant précisé la différence que je voyais entre intertexualité et interdiscursivité. L'intertexualité, c'est l'intertexte, ce qui a été effectivement dit par écrit ou oral sous une configuration déterminée, et qui donne lieu à du discours rapporté. L'interdiscursivité, c'est ce qui se dit mais qui circule sous différentes formes, et qui est repris dans la formulation que choisit le sujet qui reprend ces divers propos. C'est ce qui finit par construire des imaginaires sociodiscursifs à travers un processus de représentations sociales. Mais pas plus chez Genette que chez Bakhtine, il n'y a la prise en compte de ce qui structure conceptuellement le lieu dans lequel va se faire la production discursive. Alors que, y compris dans la conversation quotidienne, il y a toujours un contrat sous-jacent, car sans sa présence, les individus ne pourraient s'entendre.

Mots. Les langages du politique : Vous accordez une place importante au concept de stratégie discursive en n'accordant, à la différence de nombreux autres chercheurs, qu'un rôle secondaire aux thèmes ou aux contenus des discours. Peut-on rendre compte de l'influence d'un discours sans s'y intéresser? Patrick Charaudeau : Il y a deux façons d'approcher le thème. Celle, assez classique, de l'analyse de contenu que pratiquent les sociologues et les historiens, et celle que moi-même je ne pratique pas, à savoir le traitement statistique des corpus, dont je ne nie pas l'utilité. Pour moi, il y a trois dimensions dans la production discursive. Une dimension situationnelle (le contrat), une dimension énonciative (la mise en scène langagière) et une dimension topicalisante, qui est de l'ordre de la sémantique des contenus. Le contenu sémantique est dans 
les mots, les formules, les types de phrases récurrentes, mais au service de stratégies qui agissent sur la dimension situationnelle (par exemple pour renforcer la légitimité de parole), sur la dimension énonciative à l'aide des possibilités de modalisation du langage (par exemple, la possibilité de manifester accord ou désaccord, etc.), et sur la dimension topicalisante par le choix des mots, des expressions et des tournures qui sont porteurs d'imaginaires et qui sont employés en fonction d'une visée stratégique. Tout cela, c'est du sens. La thématique, pour ce qui est de son sens, se trouve donc traitée en rapport avec les dimensions situationnelle et énonciative.

Mots. Les langages du politique : La centration sur les stratégies discursives semble contribuer à redéfinir la notion de corpus dans la mesure où l'on s'intéresse davantage à de grandes logiques de discours qu'à des matériaux langagiers spécifiques bien circonscrits. Pouvez-vous préciser la place et les modes d'établissement du corpus dans vos derniers travaux?

Patrick Charaudeau : Il y a plusieurs types de corpus. Il y a des corpus linguistiques, ce que j'ai pratiqué quand je décrivais les systèmes et les lexiques d'une langue. Il y a des corpus textuels, ensemble de textes écrits ou oraux, quand on analyse des productions littéraires ou non littéraires. Il y a des corpus hypertextuels, au sens de Genette, quand on rassemble, par exemple, des Unes de journaux. Il y a aussi des corpus de mots, proches des corpus linguistiques, qui rassemblent les récurrences de tel ou tel terme en s'appuyant sur des outils informatiques et en prenant en compte les contextes (à partir, par exemple, de la base Frantext). Pour ce qui me concerne, je construis les corpus au coup par coup, autant que de besoin, car la notion de discours - notion ambivalente s'il en fut, car elle se confond tantôt avec « texte », tantôt avec des «procédés» (descriptif, narratif, etc.), tantôt avec ce que Benveniste appelle la signifiance, c'est-à-dire les systèmes de sens qui courent sous la manifestation discursive - intègre ces différentes dimensions.

Dans mon dernier ouvrage sur la Conquête du pouvoir (2013), je me suis intéressé à ce que j'ai appelé la matrice des discours de droite et de gauche. Dans le premier, court l'imaginaire de la mère-nature qui s'impose à l'homme; dans le second, c'est l'imaginaire du progrès qui veut que ce soit l'homme qui, par la culture, impose sa loi à la nature. Ce n'est pas sur la base d'un seul et même corpus que j'ai pu mettre cela en évidence. C'est en traversant divers types de discours différents les uns des autres, et en cherchant moins les récurrences que les constantes d'ordre sémantique. À l'inverse, si on analyse des slogans politiques, par exemple les slogans qui s'exposèrent lors de la «Manif pour tous », dans ce cas, on a affaire à un corpus au sens classique.

Cette démarche justifie d'ailleurs l'emploi que je fais de l'expression phénoménologie du discours, qui est complétée par une démarche herméneutique. L'herméneutique étant un travail d'interprétation constant et critique, 
on doit également se placer du côté de celui qui reçoit, interprète ou analyse puisque, une fois de plus, le sens est le résultat d'une coconstruction. Si j’insiste tant sur la différence entre effets visés et effets produits, c'est pour rappeler cette dissymétrie de l'acte de communication qui fait qu'il n'y a pas nécessairement de coïncidence entre la production et la réception-interprétation de l'acte de communication. Pour moi, une analyse ne peut jamais conclure à un sens, mais à des sens possibles, à ce que j'appelle des possibles interprétatifs.

Pour conclure sur la question du corpus, je voudrais dire quelque chose concernant ce que l'on appelle les grands corpus et leur traitement statistique. Ils sont très utiles. La question est de savoir ce que l'on en fait. On veut trop souvent en faire la clef des effets d'influence. Mais la récurrence d'un mot ou d'une expression n'est pas le gage de son impact. Pour ce qui me concerne, j'utilise la notion de mot-symptôme. Le mot-symptôme (comme j'ai été conduit à parler d'image-symptôme à propos du traitement par les médias des événements du 11 septembre) est un mot qui est chargé sémantiquement par le contexte discursif dans lequel il est employé et par la situation dans laquelle il surgit. C'est le cas de l'expression «fracture sociale» dont s'est emparé Jacques Chirac, expression semble-t-il empruntée à Emmanuel Todd. En fait, Jacques Chirac ne l'employa qu'une seule fois, mais elle eut un impact immédiat du fait que les médias la répercutèrent. Si, donc, il y a eu un emploi récurrent, c'est dû à la diffusion médiatique, ce que des études lexicométriques peuvent très bien mettre en évidence. Et c'est donc utile de le savoir. Mais ce que je veux dire, c'est que la force de l'expression et de son effet tient davantage à ce que cette expression rappelle d'autres emplois de «fracture » en rapport avec "société » (intertextualité), lesquels se trouvent plutôt dans les discours de gauche, dans une situation particulière, la campagne électorale de 1995, qui est un moment d'enjeu important où il s'agit pour la droite de faire des clins d'œil à l'électorat de gauche. C'est pris dans un certain mode de circulation de la parole dans l'espace public qu'un mot ou une expression finit par avoir un certain impact. Sous les mots, il y a toujours, d'une façon ou d'une autre, du politique. Et la raison n'en est pas nécessairement statistique.

Mots. Les langages du politique : Un autre concept important dans votre théorisation est celui d'imaginaire sociodiscursif. C'est un concept sur lequel vous insistez et pour lequel vous semblez indiquer votre préférence en lieu et place d'idéologie, alors que ce dernier concept est déterminant dans des approches critiques comme celle de Teun van Dijk'1. Comment situer votre propre approche au sein de l'offre théorique en la matière? Peut-on faire l'économie de ce concept d'idéologie pour rendre compte en particulier des positionnements politiques et des différents discours qu'ils génèrent dans l'espace public?

1. Voir en particulier Teun A. Van Dijk, 1998. 
Patrick Charaudeau : C'est vrai, j'ai eu tendance à vouloir désidéologiser les discours tout en reconnaissant que le discours est, en revanche, toujours axiologisé : ce n'est pas la même chose qu'idéologisé. Axiologisé veut dire qu'il n'y a pas de discours neutre, qu'il y a des polarisations, qu'il y a des valeurs. Et tout cela dépend de la façon dont se construisent les discours à travers le processus des représentations sociales partagées...

Il faut remonter à Destutt de Tracy qui créa le terme d'idéologie en lui donnant un sens très général d'ensemble d'idées et de pensées construites de façon cohérente et propre à des groupes sociaux. Il s'agit donc de «systèmes de pensée », de "systèmes de signifiance», qui sont autant de façons d'interpréter le monde. Ce qui m'a toujours gêné est que cette notion soit surtout employée pour des systèmes de pensée politique (ce qu'en ont fait Marx et d'autres). Pour moi, c'était réducteur. C'est pour cela que j’ai proposé, entre autres, de remplacer le concept d'idéologie par celui d'imaginaire sociodiscursif, qui me paraît beaucoup plus ouvert, et pouvoir rendre compte de beaucoup de systèmes de pensée qui ne sont pas encadrés, encastrés dans des systèmes politiques, et qui sont bien répertoriés dans l'Histoire et la Philosophie politiques. Parallèlement à cela, il y a ce que j'ai découvert en Amérique latine, à savoir l'influence qu'avait l'analyse critique du discours, promue par Van Dijk (il ne faut pas oublier que c'est Norman Fairclough qui est à l'origine de l'analyse critique du discours). Là, les chercheurs et universitaires latino-américains, très sensibilisés, et à juste titre, par les problèmes d'ordre politique, de rapports de force, de pouvoir et de soumission des citoyens au pouvoir des élites, se sont précipités sur l'analyse critique du discours en en faisant ce qu'on faisait en France dans les années soixante-dix, à savoir l'étude des discours des différents partis et acteurs politiques. Or, il me semblait que cela ne rendait pas compte du fait que les imaginaires sociodiscursifs témoins des rapports de force ne sont pas à sens unique, ne sont pas univoques, et permettent des alternances de positions de pouvoir. Pour moi, les imaginaires sociodiscursifs sont toujours rapportés à des groupes sociaux. Comme je l'ai dit tout à l'heure, j'ai, dans mon dernier livre sur la Conquête du pouvoir, décrit ce qui m'apparaissait être l'imaginaire sociodiscursif matriciel de la droite, et l'imaginaire sociodiscursif matriciel de la gauche. Cela permet de constater que, à l'intérieur même de ces imaginaires matriciels, on peut jouer, créer des variantes, ce qui fait que l'imaginaire bénéficie d'une certaine souplesse. Et ce, à l'encontre de l'idéologie qui, elle, a un caractère figé. En fait, on peut dire que l'idéologie est un imaginaire qui se durcit, et de ce point de vue, on peut continuer à parler d'idéologie lorsqu'il s'agit d'un imaginaire sociodiscursif qui se fige. Autrement dit - discursivement et non point philosophiquement-, l'imaginaire sociodiscursif est une notion générique qui témoigne d'un certain système de croyance, à l'intérieur duquel on peut trouver des discours idéologiques qui s'essentialisent : les -ismes (égalitarismes, racisme, antisémitisme) sont en 
quelque sorte des concrétions d'imaginaires qui circulent dans les groupes sociaux à propos de visions sociales, d'imaginaires, sur la façon de concevoir les relations entre les individus de la Cité, les distinctions de classe, de race, de religion. Je l'ai expliqué dans Le discours politique. Les masques du pouvoir (2005). Mais je reconnais que ce sont des notions bien difficiles à définir. D’abord, parce que, jusque dans les années quatre-vingt-dix, la philosophie et les sciences sociales ont été dominées par la conception marxiste qui voit la conscience sociale aliénée par des idéologies dominantes. Puis, avec entre autres la critique de Ricœur (1997) - qu'il faut relire -, qui met en cause la partition sociale du « réel », la question est reprise en termes de «systèmes de croyances sociales» (Van Dijk), d' «imaginaires sociaux» (Castoriadis), ce qui fait qu'on ne peut plus dire qu'il y ait consensus sur cette notion. Ensuite, parce que dans l'usage qui en est fait en politique, c'est toujours l'adversaire qui est taxé d'idéologue (la droite vis-à-vis de la gauche et inversement), à qui on reproche son idéologie, sans que l'on sache vraiment de quoi il s'agit.

En fait, les individus qui vivent en société sont à la fois producteurs d'imaginaires sociaux par les discours qu'ils produisent, et prisonniers de ceux-ci. Mais ils en sont plus ou moins conscients et se battent pour les revendiquer ou les rejeter, selon les enjeux de communication dans lesquels ils se trouvent. C'est donc l'exploitation des imaginaires sociaux dans des circonstances historiques données qui produit l'idéologie, laquelle cherche toujours à dénoncer des rapports de domination et de soumission univoques. Entendons-nous bien : la vie sociale est marquée par des rapports de force et de domination, mais ceux-ci sont divers, multiples et non nécessairement univoques, car ils peuvent s'inverser. Et donc la question, pour un analyste des discours, du moins pour moi, est d'essayer de déterminer les imaginaires prégnants dans telle société, à telle époque, sans a priori sur ces rapports de force, quitte à voir comment ils surgissent, comment ils s’idéologisent.

Mots. Les langages du politique : Des chercheurs inscrits dans les sciences politiques s'intéressent au discours politique en empruntant parfois des outils et des concepts aux sciences du langage. Quelle est à votre avis la place spécifique de l'analyse du discours dans cet espace de recherches?

Patrick Charaudeau : Le plus important pour moi, c'est la problématique de l'interdisciplinarité. J'ai organisé là-dessus une journée d'étude au CNRS avec des biologistes à propos des questions qui se posent autour de la notion de race, et j'ai aussi lancé un débat dans la revue Questions de communication (les numéros 17, 18 et 19)² autour de l'idée d'une interdisciplinarité focalisée. Des chercheurs appartenant à diverses disciplines (philosophie, sociologie,

2. 〈http://questionsdecommunication.revues.org/210〉, 〈http://questionsdecommunication. revues.org/281〉 et 〈http://questionsdecommunication.revues.org/328〉, 
anthropologie, linguistique, histoire) ont réagi et on a pu ainsi dialoguer. J'ai une position très volontariste sur l'interdisciplinarité, que j'oppose à la pluridisciplinarité qui n'est qu'une juxtaposition de disciplines, alors que l'interdisciplinarité suppose qu'on examine les notions que traitent d'autres disciplines, comment elles les définissent, pour considérer dans quelle mesure on peut réutiliser ces définitions dans son propre champ disciplinaire, mais en précisant où on les prend et ce qu'on en fait. Pour ma part, c'est ce que je me suis employé à faire avec les notions de «représentations sociales », d' «identité» et de «stratégie».

On peut donc faire de l'interdisciplinarité autour des notions, mais aussi en aval, c'est-à-dire au moment de l'interprétation. Par exemple, concernant le discours politique, il est nécessaire d'aller voir ce qui se dit en philosophie politique, en sociologie politique, en histoire, pour étendre l'interprétation au-delà des résultats proprement discursifs. Je me trouve à l'heure actuelle dans un laboratoire dit interdisciplinaire (Laboratoire de Communication et Politique du CNRS), et je constate en permanence la difficulté qu'il y a à s'entendre entre historiens, sociologues et politistes, parce que les concepts (y compris quand on emploie parfois les mêmes termes) et les modes de raisonnement qui dépendent des méthodologies de chaque discipline ne sont pas les mêmes. Je constate, par exemple (on devrait en faire une recension), que dans les colloques organisés par des sociologues ou des chercheurs de sciences politiques, il y a rarement des chercheurs en sciences du langage. Je me suis même heurté (gentiment), lors d'une soutenance de thèse pluridisciplinaire, à un collègue de sciences politiques qui ne comprenait pas que les sciences du langage s'occupent du fait politique, comme si le champ était réservé. Donc, dans les faits, on est bien loin d'une véritable interdisciplinarité. Alors, dire que les sociologues s'inspirent de l'analyse du discours, là je suis extrêmement sceptique, d'autant que j'ai cru comprendre qu'en ce moment, la sociologie est en crise entre divers courants. Heureusement, il y a des rencontres entre chercheurs (personnellement, je les suscite) et des lieux de rencontre (des ouvrages collectifs). C'est ce à quoi s'emploie la revue Mots. Les langages du politique. Mais il faut continuer le combat, parce que, me semblet-il, l'interdisciplinarité est ce qui évite ce que j'appelle l' "arrogance » disciplinaire, c'est-à-dire l'enfermement dans une seule discipline pensant qu'elle est seul maître de l'objet d'étude. Il n'y a pas d'objet exclusif d'une discipline.

\section{Mots. Les langages du politique : Nous parlions surtout de politologues...}

Patrick Charaudeau : Il y a des politologues - j'en ai fait l'expérience - qui ont du mal à comprendre que l'on puisse étudier le phénomène politique autrement que dans le cadre des sciences politiques. Dans mon dernier livre, La conquête du pouvoir, j'ai fait une analyse critique des sondages en montrant comment, si l'on s'intéresse aux sondages par le langage, tout est piégé. Il est 
nécessaire de distinguer divers types de sondages : d'intention, de préférence, d'évaluation, car chacune de ces catégories dit quelque chose de particulier quant à l'opinion exprimée. J'ai montré comment les sondages d'évaluation sont trompeurs et auto-réalisants. Mais je n'ai vu nulle part que cette proposition d'analyse soit reprise et discutée. De plus, je crois qu'il y a un malentendu sur ce que l'on appelle un politologue. Il faudrait distinguer politologue et politiste : celui qui est sollicité par les médias pour commenter l'actualité et celui qui travaille à analyser le phénomène politique de façon plus approfondie.

Mots. Les langages du politique : Vos derniers ouvrages (Charaudeau, 2008a, $2008 \mathrm{~b}, 2013)$ sur le discours politique contrastent avec vos productions précédentes, puisqu'ils s'adressent à un plus large public et en étant liés à l'actualité. N'y a-t-il pas un risque pour le chercheur dans ce type de tentative, en particulier celui de glisser vers un positionnement d'essayiste?

Patrick Charaudeau : Là-dessus, j'ai deux choses à dire. D'une part, ce que sont les contraintes éditoriales. On ne peut que constater que les écrits qui sont acceptés par les maisons d'édition et commentés par les médias sont ceux qui se présentent, au mieux comme des essais, au pire comme des pamphlets (voir le succès du dernier livre d’Éric Zemmour). Le pamphlet est un genre répertorié (bien défini par Marc Angenot, 1982)3 qui est destiné à engendrer la polémique. Et, comme on le sait, la polémique n'est pas ce qui aide à penser. L'essai, lui, peut avoir diverses couleurs. Qu'il soit critique est bien, c'est d'ailleurs là son rôle. Mais s'il est dénonciateur, alors il devient un pamphlet et l'on ne sait plus trop quoi en dire. Mais c'est à l'heure actuelle ce qui circule le mieux, ce qui domine, dans l'espace public, ce qui laisse peu de place pour des écrits d'analyse approfondie. Moi, j'aurai toujours du mal à écrire comme cela.

Pour ce qui me concerne, le seul de mes ouvrages que j'ai voulu grand public est le Petit traité de politique à l'usage du citoyen (2008a) et peut-être aussi Entre populisme et peopolisme. Comment Sarkozy a gagné! (2008b) Encore que pour ce dernier, il y a des passages qui impliquent que l'on ait une certaine connaissance des procédés de l'analyse du discours. Mais les autres, non. C'est que personnellement, j'ai du mal à expliquer quelque chose sans me référer à des outils d'analyse parce que c'est, me semble-t-il, le rôle du chercheur. Alors, évidemment, ça ne colle pas avec les contraintes éditoriales qui tiennent à l'idée que les maisons d'édition se font du lectorat. C'est pour moi une question d'éthique. L'éthique qui dit que le chercheur n'analyse pas pour faire plaisir mais pour faire comprendre. Je disais tout à l'heure que le projet phénoménologique d'analyse doit être complété par un projet herméneutique. C'est, me semble-t-il, ce qui doit caractériser la démarche des sciences humaines et sociales. C'est la différence qu'il y a pour moi entre un discours

3. Voir également Hastings et al., 2009. 
dénonciateur, pamphlétaire (le pamphlétaire, c'est critiquer vertement en se moquant et en cherchant à disqualifier les idées contraires), et un discours explicatif qui consiste à interroger plus qu'à affirmer. C'est ce que j'ai fait à la fin de La conquête du pouvoir. La réflexion finale est présentée sous forme interrogative : est-ce que le politique doit répondre à la demande sociale ? Estce qu'une démocratie participative est possible? Tout cela sur le fond d'une hypothèse qui est que le discours populiste est en train de recycler les discours extrêmes de droite et de gauche, ce qui expliquerait le désarroi de l'électorat. Donc, ce n'est pas un essai.

Mots. Les langages du politique : Dans votre traitement de la question de la laïcité exposé dans le dernier ouvrage que vous avez dirigé (Charaudeau, 2015), vous vous êtes plus spécialement intéressé à la notion de controverse. En quoi est-elle différente de celle de débat ou encore de polémique (examinée récemment par Ruth Amossy : voir Oger, 2015)?

Patrick Charaudeau : Ces distinctions vont être présentées dans un ouvrage que je suis en train d'écrire sur la controverse. La polémique n'est pas un genre, ni un contrat de communication. On ne décide pas que l'on va passer un contrat polémique dans un échange privé ou public. En revanche, on peut décider d'avoir, stratégiquement, un comportement polémique. La controverse, elle, relève du genre. On repère un problème de société, on décide de mettre en face-à-face des personnes qui ont un point de vue différent autour d'une certaine problématique, comme c'est le cas de la laïcité, et on attend des partenaires de l'échange qu'ils exposent leur point de vue de façon argumentée. Cela devient une controverse. La polémique est une stratégie qui peut traverser divers genres. Elle n'est pas un genre en soi. Vous savez que je définis le genre discursif, non pas par les récurrences discursives des textes, mais à partir de la notion de contrat, c'est-à-dire de ce qui détermine les conditions situationnelles de l'acte de communication.

De fait, si quelque chose peut être utile à la démocratie, ce sont bien les controverses, et non point la polémique qui est un obstacle au développement de la pensée. Dans l'ouvrage que je prépare là-dessus, je typologise les situations d'interaction verbale. Et parmi celles de confrontation, j'établis une différence entre discussion, controverse, débat et je mets à part tout ce qui est de l'ordre de la querelle, de la dispute (qui n'est pas la disputatio de la rhétorique), et donc la polémique.

La discussion est un contrat avec une certaine distribution des rôles de parole, tantôt descriptifs, tantôt narratifs, tantôt argumentatifs : ce peut être le cas d'une conversation entre amis, d'un échange de points de vue sur un film, sur une question administrative dans une réunion de travail, ou d'une discussion scientifique, etc. La discussion n'est pas une dispute. Et contrairement à ce que disent certains, la dispute n'est pas un genre, elle ne relève pas d'un 
contrat. Il peut se trouver que, dans une discussion, on finisse par avoir une dispute à cause de ce que disent les uns et les autres, et qu'au lieu de prendre en compte l'argument adverse on se cantonne sur une position, comme on dit «sans discussion». Le genre discussion est une sorte de genre générique.

La controverse, en revanche, est un genre qui obéit à un contrat. Est posé un problème qui fait l'objet d'un certain questionnement (une problématisation), qui engendre des points de vue opposés et exige que soit développée une argumentation pour les étayer, sans que, a priori, on cherche à mettre en cause la personne du débatteur opposé. Dans la controverse, on cherche à discuter de l'objet mis en questionnement. Par exemple, à propos de la laïcité : «Fautil interdire le voile à l'école secondaire?»; «Faut-il exclure les jeunes filles voilées?» (On peut se reporter à l'ouvrage sur la laïcité pour en voir les divers questionnements). La controverse est toujours tendue vers une recherche de la vérité; la polémique vers une recherche de triomphe sur l'adversaire.

La notion qui pose problème est celle de débat. Le débat a lui aussi vocation à être une notion générique. Sa particularité est qu'au lieu d'être centré sur un seul questionnement, il se disperse sur plusieurs. De plus, dans un débat, il y a de nombreux protagonistes, chacun tirant dans la direction qui lui convient et brouillant les questionnements. C'est ce qui se passe dans les débats télévisés. Dans un débat, il peut y avoir des moments de controverse qui se centrent sur une problématique particulière avec échange d'arguments, et des moments de polémiques dans lesquels les participants se mettent en cause les uns les autres.

Dans tous ces cas, on croise la notion de dispositif. On peut concevoir cette notion de deux façons. D'une façon conceptuelle, dans la mesure où tout contrat, pour moi, est organisé autour d'un dispositif qui met en présence des instances de communication (par exemple, l'instance politique, l'instance citoyenne et l'instance de médiation) et détermine l'enjeu qui les relie. Mais on peut concevoir cette notion de façon plus formelle : ce que serait un face-à-face dans un débat télévisé, une interview à la radio, un meeting politique, une réunion de travail quelconque, etc. Dans les discussions, controverses et débats, c'est cette deuxième définition qui est pertinente, car une discussion ou une controverse peut faire l'objet de divers dispositifs formels. La polémique, en revanche, n'a rien à voir avec un dispositif parce qu'elle peut traverser n'importe lequel d'entre eux. La dispute non plus n'est pas un dispositif. Ce sont des façons de parler qui sont de l'ordre de la stratégie dont l'essentiel est la mise en cause de la personne, la mise en cause de la position de l'autre - lequel, alors, passe du statut de simple opposant à celui d'adversaire. La stratégie, pour moi, a un sens très précis : c'est la marge de manœuvre que laisse le contrat et dans laquelle peut s'immiscer le sujet parlant pour se singulariser, s’individuer. La stratégie, c'est la possibilité que peut avoir le sujet de jouer plus personnellement à l'intérieur des instructions imposées par le contrat de communication. 
La polémique est donc le procédé par lequel l'autre est mis en cause en tant que locuteur, en tant que personne aussi bien dans son identité sociale, psychologique, que discursive, c'est-à-dire sa façon de parler. On en trouvera des exemples dans l'analyse que j'ai faite du débat de la présidentielle de 2012 (paru dans la revue Langage \& Société, nº146, 2013b).

Mots. Les langages du politique : Votre étude du discours populiste vous conduit à rapprocher dans vos derniers ouvrages des types de discours (extrême droite et extrême gauche) posés habituellement comme antagonistes. Pouvezvous revenir sur cette analyse qui est de nature à surprendre si l'on considère par exemple, dans la dernière période, ce que sont les positions respectives de ces deux camps face à la question des migrants?

Patrick Charaudeau : Oui, parce que c'est un phénomène nouveau de nos démocraties occidentales travaillées par la mondialisation, la crise économico-sociale et les mouvements migratoires, sur fond de disparition de l'antagonisme entre projet de société communiste et projet de société capitaliste, avec la chute du mur de Berlin. Le discours populiste joue avec trois imaginaires sociaux : celui de la déchéance sociale et de ses causes qui se trouvent dans un bouc émissaire; celui du leader providentiel qui est censé être le seul à pouvoir remettre la société en état; celui d'un projet de société comportant des valeurs (souvent retour de valeurs perdues) susceptibles de relever le pays. Dès lors, on retrouve dans le discours populiste les thématiques du discours politique général, mais poussées à l'extrême : stigmatisation de l'ennemi extérieur ou intérieur (bouc émissaire) ; rôle protecteur d'un État qui serait en prise directe avec le peuple et dont le leader est le garant; retour des valeurs identitaires. Or, on constate que les discours de l'extrême droite et de l'extrême gauche ont des points communs, même si parfois sont utilisés des termes différents : ils décrient l'ensemble de la classe politique et désignent les élites comme l'ennemi à combattre ; ils prônent un État puissant et protecteur, redistributeur des richesses; ils disent se préoccuper de ce qu'à droite on nomme «le petit peuple» et à gauche la «classe populaire»; ils glorifient un leader capable de faire le coup de force et de «renverser la table». Évidemment, il y a quelques valeurs sociétales qui les distinguent, parce que l'extrême droite reste sur la vision d'une société hiérarchisée selon l'ordre de la nature, et l'extrême gauche sur la vision d'une société égalitaire et solidaire. Mais l'habillage discursif populiste de ces visions de société (par exemple, dans la dernière campagne présidentielle, les tactiques de disqualification outrancière de l'adversaire - l'une des caractéristiques du discours populiste - furent employées des deux côtés, et même avec plus de virulence par Jean-Luc Mélenchon qui traita Marine Le Pen de «chauve-souris», "semi-démente», «yeti de la politique»), cet habillage populiste gomme ce qui les oppose auprès d'un électorat fragile qui n'a plus guère de points de repère. C'est ce qui permet d'expliquer le glissement d'une partie de l'électorat ouvriériste et de la classe moyenne vers le Front national. 
Je crois qu'il faut se débarrasser de l'idée que l'électorat, c'est l'opinion publique. L'électorat est un sous-ensemble fragmenté de l'opinion publique, si tant est qu'il y aurait une opinion publique homogène. Chacune de ses catégories est imprégnée des valeurs que défend telle ou telle partie de l'opinion publique, mais la conduite de chacune d'elles est guidée parl'acte symbolique qu'est le vote, motivé par autant de passion que de raison. Ce qui fait que les discours produits par les acteurs politiques sont recyclés par l'électorat à l'aune de son idiosyncrasie. Chacune de ses catégories ne retient que ce que lui commande celle-ci. Donc, pour répondre à la question des migrants, je constate qu'un certain discours populiste - par exemple, le sécuritaire qui agite le fantôme du «migrant envahisseur » - recycle à la fois les discours de droite et de gauche. Bien sûr, il y a, à ce sujet, une différence entre eux : la droite reprend explicitement le thème du bouc émissaire migrant; la gauche se veut plus compréhensive. Mais dans la mesure où celle-ci ne produit pas un discours radicalement différent, nettement compassionnel et structurant sur la politique à mener vis-à-vis des migrants, se contentant de faire la distinction entre réfugiés et migrants économiques, elle n'est pas audible. Les discours de gauche s'attachent moins à défendre le multiculturalisme (je ne parle pas de tel ou tel intellectuel ou groupe militant) qu'à prôner l'intégration au nom de la solidarité sociale et des valeurs universelles. Mais on voit bien, à travers ces discours, que la gauche est mal à l'aise devant les revendications de son propre électorat qui parle en termes de menace, d'envahissement ( $40 \%$ des sympathisants de gauche disent qu' «il y a trop d'étrangers en France») et d'assistanat injustifié. Au fond, ce qui domine à gauche, pour tenter de satisfaire cet électorat, est une revendication nationaliste, à l'instar de Jean-Luc Mélenchon qui se dit «populiste patriote». Une fois de plus, je ne dis pas que, idéologiquement, la droite et la gauche sont équivalentes. Je dis que leur discours produit auprès de l'électorat un effet de brouillage des oppositions et donc un recyclage discursif qui fait que la gauche n'a plus l'assentiment populaire et que c'est le Front national - et la droite classique - qui se l'est approprié. C'est pourquoi j'avance l'hypothèse que les partis extrêmes sont conduits, par l'usage de certains des mêmes ingrédients du discours populiste, à se « désextrémiser» tout en restant « radicaux».

\section{Références}

ANGENOT Marc, 1982, La parole pamphlétaire. Typologie des discours modernes, Paris, Payot.

Bourdieu Pierre, 1982, Ce que parlerveut dire, Paris, Fayard.

Charaudeau Patrick, 2005, Le discours politique. Les masques du pouvoir, Paris, Vuibert.

- 2008a, Petit traité de politique à l'usage du citoyen, Paris, Vuibert. 
- 2008b, Entre populisme et peopolisme. Comment Sarkozy a gagné!, Paris, Vuibert.

- 2013a, La conquête du pouvoir. Opinion, persuasion, valeurs, les discours d'une nouvelle donne politique, Paris, L'Harmattan.

- 2013b, «L'arme cinglante de l'ironie et de la raillerie dans le débat présidentiel de 2012 », Langage et société, nº 146.

- 2015, La laïcité dans l'arène médiatique. Cartographie d'une controverse sociale, Paris, INA. Hastings Michel, PASSARd Cédric, Rennes Juliette éd., 2009, Mots. Les langages du politique, nº 91 , Que devient le pamphlet?

OGER Claire, 2015, "La polémique est un mode de gestion socio-discursif du conflit dans les sociétés démocratiques», Entretien avec Ruth Amossy, Mots. Les langages du politique, n ${ }^{108}$, Thèmes et thématiques dans le discours politique.

Ricceur Paul, 1997, L'idéologie et l'utopie, Paris, Le Seuil.

VAN DIJK Teun A., 1998, Ideology. A Multidisciplinary Approach, Londres, Sage. 
